\title{
Experimental Investigations into the Use of Piezoelectric Film Transducers to Determine Particle Size through Impact Analysis
}

\author{
James Robert Coombes \\ School of Engineering and Digital Arts, University of Kent \\ Canterbury, Kent CT2 7NT, UK \\ jrc55@kent.ac.uk
}

\author{
Yong Yan \\ School of Engineering and Digital Arts, University of Kent \\ Canterbury, Kent CT2 7NT, UK \\ y.yan@kent.ac.uk
}

\begin{abstract}
Sensors are required to determine the particle size of granular materials in a variety of industries such as energy, chemical manufacturing and food processing. The importance of accurately monitoring the particle size is essential in quality control in these industrial sectors. This paper presents the use of a custom made piezoelectric PVDF film transducer that is capable of determining the particle size of granular material through impact analysis. Experiments were carried out using a purpose-built test rig using ball bearings of different sizes traveling at different velocities. Through power spectral analysis of the impact signal it has been determined that different components of the signal spectrum may relate to different characteristics of the particle itself such as particle impact velocity and particle size. A comparison between the experimental data and system modelling results using the known mechanical characteristics of the test particles shows close similarities.
\end{abstract}

Keywords - piezoelectric film, impact, PVDF, particle size, particle velocity

\section{INTRODUCTION}

The ability to determine the particle size in granular materials is important in many industrial sectors such as energy, chemical manufacturing and food processing. The importance of accurately determining the particle size is vital for quality control.

One of the main areas of interest is an online method for monitoring particle size distribution during the transport of granular materials. One of the most common ways granular materials are transported is through the use of bulk solid pneumatic conveying systems. This type of system uses high velocity air to convey pulverised material along a pipe using either positive or negative pressure [1].

Many sensing methods have been researched such as optical [2 and 3], electrostatic [4] and impact/acoustic [5 and 6] to determine particle size in bulk solid pneumatic conveying systems. The optical method uses a transparent window and a camera to capture an image of the conveyed particles, a laser screen is used to illuminate the particles as they travel past the camera's view. This method has the disadvantage that the transparent window is susceptible to contamination by the fine dust in the material. However, this problem was addressed by the use of an air purging system to prevent particles coming into contact with the window [3].

Electrostatic sensors have also been deployed to determine particle size. The principle of using electrostatic sensors is that as the particles travel down the pipe they pick up an electrostatic charge due to friction with each other and the air [1]. The use of electrostatic sensors to determine particle size is based on the idea that a larger particle carries more electrostatic charge on its surface than that of a smaller one of the same type [4]. However, Coombes and Yan [7] found that smaller particles had a higher level of electrostatic charge compared to larger ones of the same type for the same mass flow rate.

Particle size has also been determined by measuring the vibrations caused by particle impacts. These sensors have used accelerometers to measure the vibrations [5], piezoelectric materials [6] and piezoelectric acoustic emission sensors [8]. All these methods use an impact bar that comes into contact with the particle flow. This method has the disadvantage of the sensor being intrusive, however, in dilute phase flow (particle concentration less than $0.1 \%$ by volume [1]) this does not cause a significant problem. However, the design of the impact bar used in these sensing methods is quite large so even in a dilute phase flow multiple impacts may occur at the same time. Also the design of the impact bar and position of the sensor means that the same sized particles travelling at the same speed could impact the bar at different locations and the sensor output would be different. Off-line experimentation has also been carried out using a piezoelectric sensor to measure the impact characteristics of spherical particles [9]. This work used a large impact plate with a piezoelectric sensor mounted on the reverse side of the plate. This sensor arrangement would be unsuitable for measuring particle size in dilute phase flow since the large plate would suffer from simultaneous impacts by many particles.

This research aims to illustrate the feasibility of using a piezoelectric film sensor that is capable to determine particle size which can be adapted for online measurement of pneumatically conveyed particles. This sensor would have the 
novel feature in that it will have a very small active sensing area, thus reducing the chance of multiple impacts occurring at the same time.

\section{MEASUREMENT PRINCIPLE}

When a moving particle collides with a fixed solid surface the kinetic energy of the moving particle is converted into an impact force. Hertz was the first to pioneer this work [10]. In order to determine the impact force the particle is assumed to be a sphere. This is because many particles in industry such as coal particles in the energy industry or shot in manufacturing are close to being spherical in shape. In order to calculate the impact force the mechanical properties of the impact surface and particle have to be taken into account. The effective elastic modulus $E^{*}$ can be determined by [11]:

$$
E^{*}=2\left(\frac{1-v_{1}^{2}}{E_{1}}+\frac{1-v_{2}^{2}}{E_{2}}\right)^{-1}
$$

Where $v_{1}$ and $v_{2}$ is the Poisson's ratio of the particle and surface respectively and $E_{1}$ and $E_{2}$ Young's modulus of the particle and surface respectively. The size (radius) of the particle also has to be taken into account. The median radius $R^{*}$ of the contact surfaces is defined as [11]:

$$
R^{*}=\left(\frac{1}{R_{1}}+\frac{1}{R_{2}}\right)^{-1} \approx R_{1}
$$

Where $R_{1}$ and $R_{2}$ are the radius of the particle and surface respectively.

From the moment of collision occurs till the moment the particle velocity reduces to zero elastic deformations occur. The time (duration of impact) is determined by [11]:

$$
s=\left(\frac{225}{64} \frac{m^{2} V^{2}}{E^{* 2} R^{*}}\right)^{1 / 5}
$$

Where $m$ is the mass of the particle, $V$ is the velocity of the particle. The maximum impact force is defined by [11]:

$$
F_{\text {max }}=\left(\frac{225 m^{3} E^{* 2} R^{*} V^{6}}{144}\right)^{1 / 5}
$$

To measure the impact force of the colliding particle a piezoelectric transducer is used to measure the vibrations caused by the particle impact. Piezoelectric materials convert mechanical stress and strain into voltage or charge [12].

PVDF (Polyvinylidene Fluoride) film is a piezoelectric material which was chosen as the transducer for the impact sensor because it has: a wide frequency range $\left(0.001 \mathrm{~Hz}-10^{9}\right.$ $\mathrm{Hz}$ ), high mechanical strength and impact resistance, high voltage output (10 times higher than piezo ceramics) and it can be manufactured into complex shapes [13].

The PVDF transducer is fabricated by having a layer of PVDF film sandwiched between two metal surfaces. The metal surfaces are applied to the PVDF surface by evaporatively deposited metals such as copper, nickel, silver or gold with the metal layer being between $500-1000 \AA$ thick [13]. The active area on the PVDF transducer is determined by where the metal surfaces overlap to form an electrode as shown in Fig. 1. These electrode shapes can be fabricated using chemical etching techniques [13].

When stress is applied to the active area on the PVDF transducer the deformation changes the charge density of the material, this change causes a voltage to be generated between the two electrodes. The output voltage is directly proportional to the magnitude and frequency of the applied stress [13].

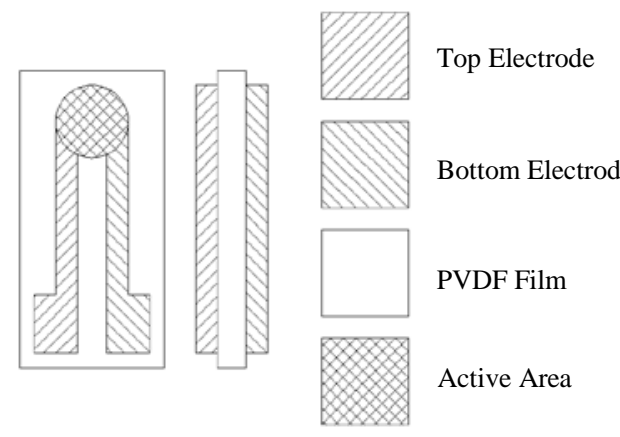

Fig. 1 Diagram of PVDF transducer

PVDF film is anisotropic which means the voltage output is dependent upon which axis the mechanical stress is applied. These piezo coefficients are most commonly used in either charge or voltage modes ( $d_{3 n}$ or $g_{3 n}$ respectively). The output of the PVDF film can be either charge or voltage. The output charge in charge mode is represented by [13]:

$$
D=\frac{Q}{A}=d_{3 n} F_{n}
$$

Where $D$ is the charge density developed, $Q$ is the charge developed $A$ is the conductive area of the electrodes, $d_{3 n}$ is the axis of the applied stress or strain and $F_{n}$ is the applied stress in the specified direction. When configured in voltage mode the output voltage is represented by [13]:

$$
V_{o}=g_{3 n} X_{n} t
$$

Where $V_{o}$ is the output voltage, $g_{3 n}$ is the axis of the applied stress or strain, $X_{n}$ is the applied stress in the specified direction and $t$ is the thickness of the PVDF film. The mechanical stress $\mathrm{X}_{\mathrm{n}}$ is calculated by:

$$
X_{n}=\frac{F_{\max }}{A}
$$

\section{IMPACT SENSOR DESIGN}

The impact sensor and data logger consists of the impact waveguide, a PVDF film transducer (illustrated in Fig. 2), a signal conditioning circuit and a microcontroller controlled analogue to digital converter (ADC) which outputs the impact sensor data to a PC or an SD memory card is shown in Fig. 3.

The waveguide primary function is to act as a conduit for the vibrations caused by the particle impact. The waveguide is secured using tightly fitting rubber washers which limits the directional axis of movement to one direction (one dimension of movement). By doing this the rubber washer also applies friction to this dimension of movement and this would reduce 
to amount of stress applied to the piezo transducer during impact. For this the output voltage is calculated by:

$$
V_{o}=\frac{g_{3 n} X_{n} t}{f_{f}}
$$

Where $f_{f}$ is the frictional force acting against the waveguide from the rubber washer during the ball bearing impact. However it is difficult to quantify the frictional force between the rubber washer due to the complexity of the surface texture of the waveguide, rubber washer and the force between the washer and waveguide [14]. For this reason the value $f_{f}$ is an estimate.

The waveguide also has a very small active sensing area (1 $\mathrm{mm}$ diameter). The advantage of this is, if the impact sensor was to be mounted in a $50 \mathrm{~mm}$ bore pipe to measure particle size on a pneumatic conveyor system, the active sensing area would be less than $0.001 \%$ of the pipe bore. This would reduce the possibility of simultaneous impacts of multiple particles. The waveguide also protects the piezo transducer from possible damage caused by particle impacts. The current sensor housing is $6 \mathrm{~mm}$ thick meaning that if the impact sensor was to be mounted inside a $50 \mathrm{~mm}$ pipe bore the nonactive sensor cross section would take up approximately $15 \%$ of the pipe bore. However, for larger bore pipes such as those in the power industry (150 $\mathrm{mm}$ and greater) this would be less of a problem.

The piezo transducer consists of a layer of PVDF film of $110 \mu \mathrm{m}$ thick with nickel copper alloy electrodes. The electrodes were fabricated via a chemical etching process using ferric chloride [13]. The piezo transducer is insulated with a layer of silicone to prevent short circuiting between the electrode and waveguide. The silicone insulation also protects the delicate electrodes. The piezo transducer is glued to the waveguide and fixed surface using a specialist adhesive for gluing silicone based rubbers as shown in Fig. 2.

The first stage of the signal conditioning circuit is a preamplifier. The preamplifier is a non-inverting amplifier (voltage mode) with the electrodes directly connected to the amplifier to reduce connection distance and maintain high signal-to-noise ratio [13]. The first stage of the signal conditioning circuit is also contained inside a shielded casing for noise protection. Having the pre-amplifier in voltage mode also has the advantage that changes in temperature have little effect on the voltage sensitivity [13].

The output of the preamplifier is then passed through an optional second-order active bandpass filter. This bandpass filter is adjustable to change the lower and upper limits of frequencies.

The signal is then digitised in an ADC. The ADC is controlled by a microcontroller using a serial peripheral interface (SPI) bus. The ADC has a 12-bit resolution and a sampling frequency of $200 \mathrm{kHz}$ (since the highest frequency expected from a PVDF film transducer in 1 directional axis will output a frequency of less than $100 \mathrm{kHz}$ [13]). Data is transmitted to a PC or saved on an SD card.

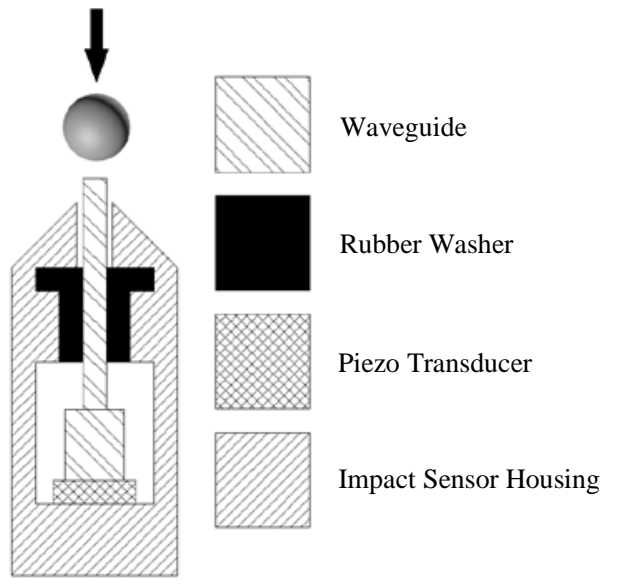

Fig. 2 Cut away view of the piezo impact sensor

\section{EXPERIMENTAL SETUP}

In order to compare the impact force measured by the impact sensor to the theoretical impact force ball bearings were used as test particles. The ball bearings are made of Delrin which is a high strength engineering polymer. Three different sizes of Delrin ball bearings were tested $2.5 \mathrm{~mm}, 3$ $\mathrm{mm}$ and $3.5 \mathrm{~mm}$ in diameter (each with a mass of $11.62 \mathrm{mg}$, $20.08 \mathrm{mg}$ and $31.88 \mathrm{mg}$ respectively).

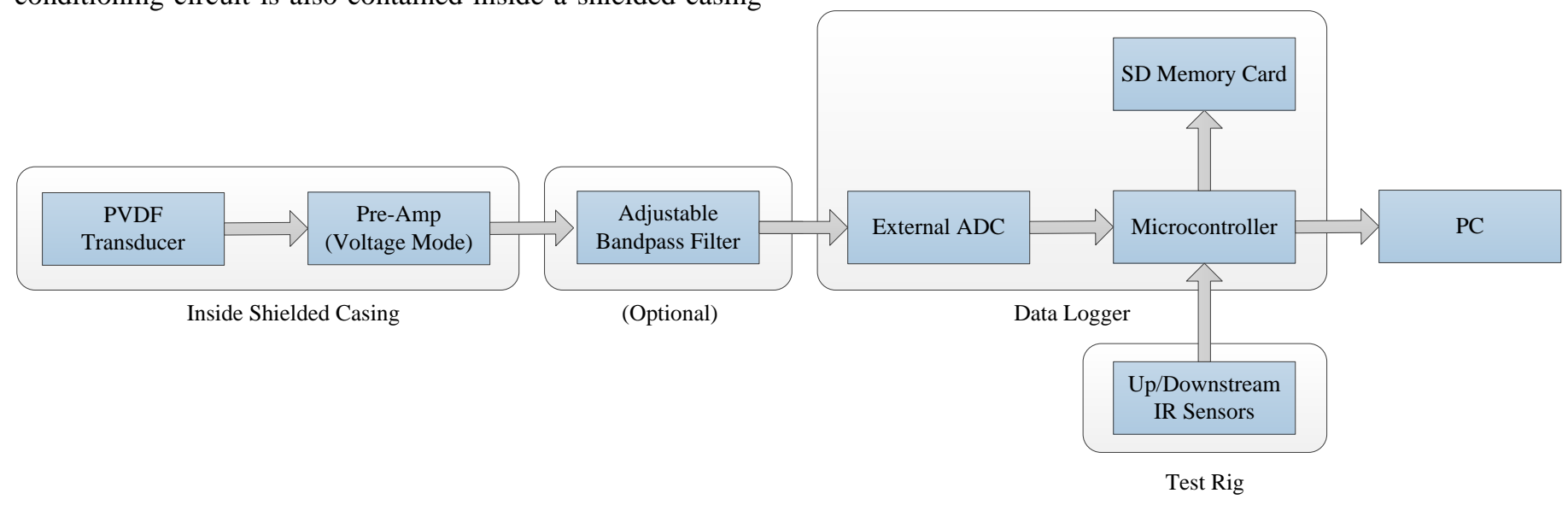

Fig. 3 Block diagram of the piezo impact sensor and data logger 
Fig. 4 shows the experimental setup used in this study. The impact sensor is rigidly mounted in a vice to prevent movement. A guiding tube is centred above the impact sensor to guide the ball bearing onto the impact sensor. The guiding tube can be adjusted to different lengths $(500 \mathrm{~mm}, 750 \mathrm{~mm}$, $1000 \mathrm{~mm}$ and $1250 \mathrm{~mm}$ ), allowing the ball bearing under test to be dropped from different heights for different impact velocities.

To independently measure the impact velocity of the ball bearing before impact at the end of the guiding tube (just before the impact sensor) optical sensors are set $25 \mathrm{~mm}$ apart as shown in Fig. 4. The optical sensors consist of an IR (infrared) emitter that creates an IR beam across the inside of the tube. This IR beam is detected by an IR receiver (IR photodiode). The two optical sensors are connected to the microcontroller as shown in Fig. 3. When the falling ball bearing breaks the upstream IR beam the microcontroller triggers the timer. When the falling ball bearing breaks the downstream IR beam the microcontroller reads the time taken for the ball bearing to travel from the upstream to the downstream sensor. The average velocity of the ball bearing is calculated from the spacing between the sensors and the measured time.

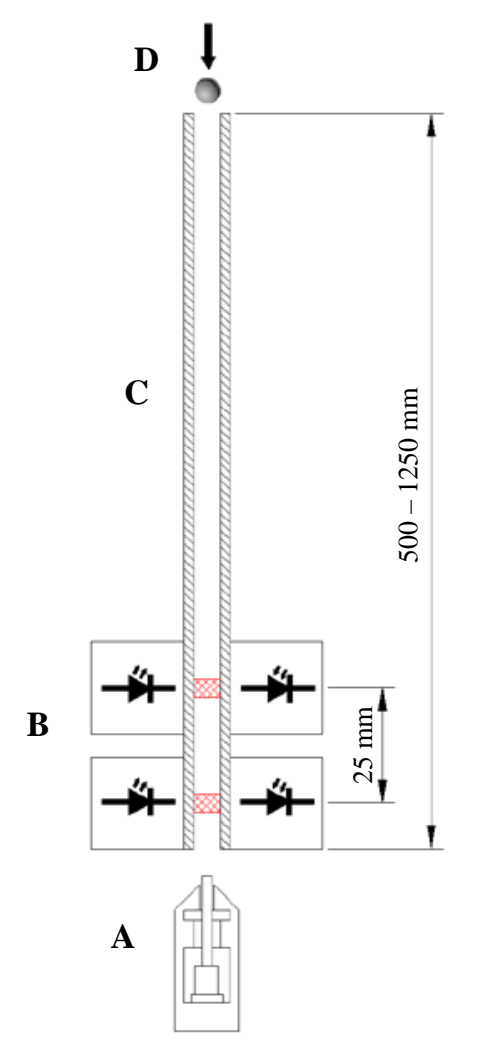

Fig. 4 Test rig (A) impact sensor, (B) upstream/downstream optical sensors, (C) guiding tube, (D) ball bearing entering guiding tube

After the downstream IR sensor has been triggered the microcontroller begins data logging from the ADC since an impact is imminent. All data from the impact sensor output is saved onto a SD memory card and an algorithm in the microcontroller determines the peak amplitude of the impact signal which is presented on the PC.

\section{RESUlTS AND DISCUSSION}

The impact sensor was first tested without the bandpass filter in order to examine the frequency component of the ball bearing impact. A total of 200 impact reading were taken for each size of ball bearings. Fig. 5 shows the peak amplitude of the impact signals. The magnitude of the unfiltered impact signals shows a high level of deviation, especially on $2.5 \mathrm{~mm}$ diameter ball bearing. The impact signals for the $3 \mathrm{~mm}$ ball bearing also show a large number of impact signals that are at the supply voltage $(1.65 \mathrm{~V})$ indicating that the preamplifier has saturated for them impacts.

Power spectral analysis of impacts of all three sizes of ball bearings is carried out for $2.2 \mathrm{~m} / \mathrm{s}, 2.4 \mathrm{~m} / \mathrm{s}$ and $2.6 \mathrm{~m} / \mathrm{s}$, as shown in Fig. 6, 7 and 8 respectively.

The power spectra in Fig. 6 - 8 indicate that the smaller diameter ball bearings have higher frequency components. Also for larger diameter ball bearings at higher velocity there is a large DC component in the signal indicating the signal has saturated to the supply voltage.

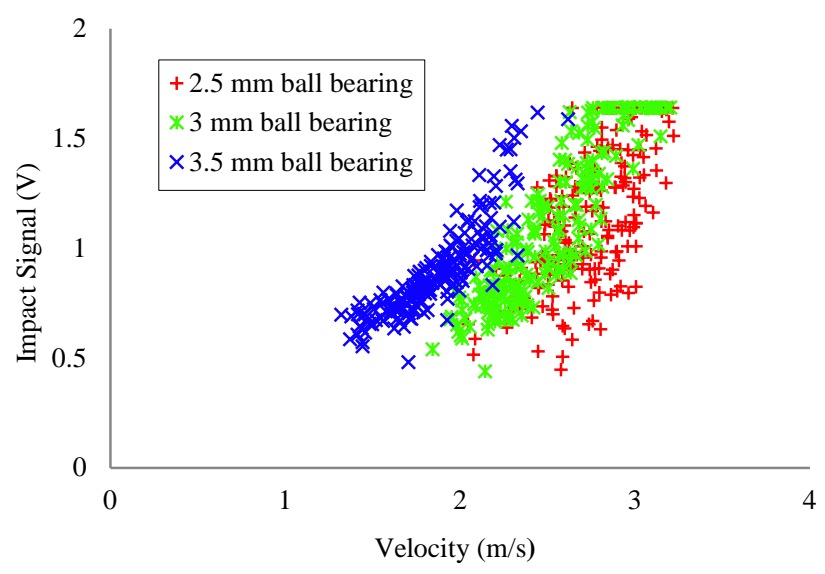

Fig. 5 Magnitude of dominant peak of ball bearing impact

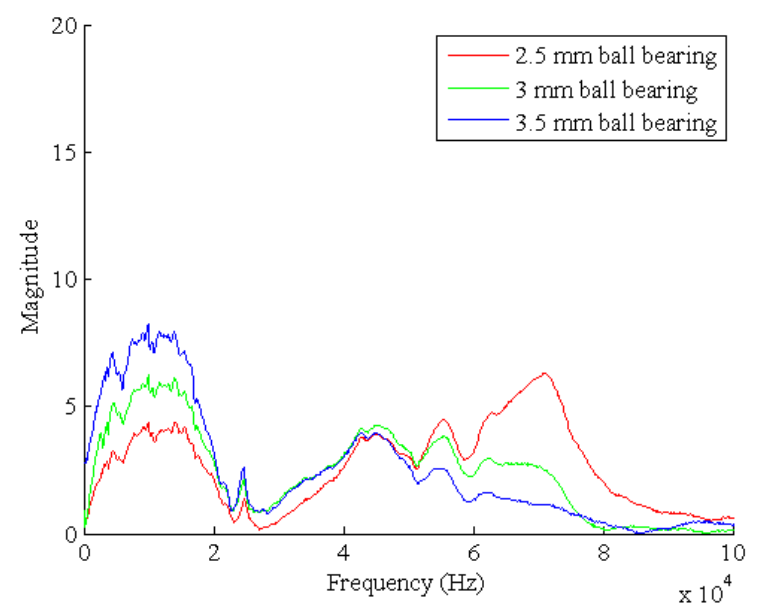

Fig. 6 Power spectra of the ball bearing impacts at $2.2 \mathrm{~m} / \mathrm{s}$ 


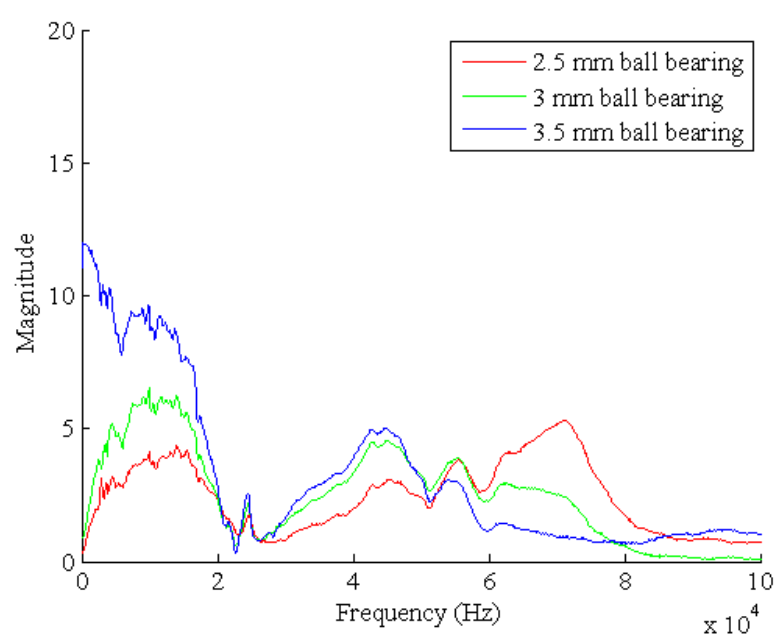

Fig. 7 Power spectra of the ball bearing impacts at $2.4 \mathrm{~m} / \mathrm{s}$

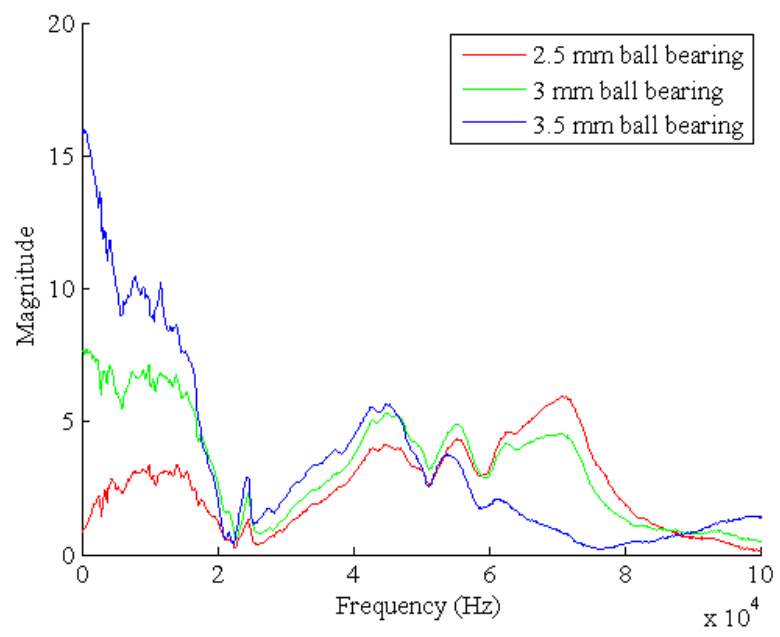

Fig. 8 Power spectra of the ball bearing impacts at $2.6 \mathrm{~m} / \mathrm{s}$

Fig. 9 - 11 shows the power spectra for the ball bearing with different impact velocities. They too show a high DC component for the higher impact velocities. For Fig. 6-11 there is a common frequency range (approximately between 5 $\mathrm{kHz}$ and $15 \mathrm{kHz}$ ) that is consistently proportional to the impact force due to a higher impact velocity or larger mass of the ball bearing.

A second order bandpass filter with a low cut-off frequency of $5 \mathrm{kHz}$ and an upper cut-off frequency of $15 \mathrm{kHz}$ was placed between the impact sensor's output and the ADC as shown in Fig. 3. This was done to remove the DC component caused by saturation and the high frequency components and only use the frequencies that were shown to be proportional to the impact force as seen in Fig. $6-11$.

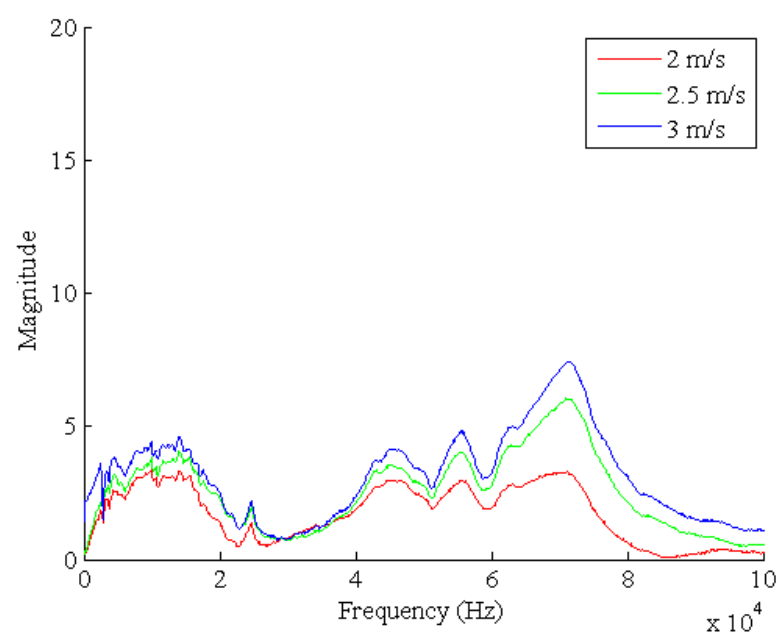

Fig. 9 Power spectra of $2.5 \mathrm{~mm}$ ball bearing impact signal

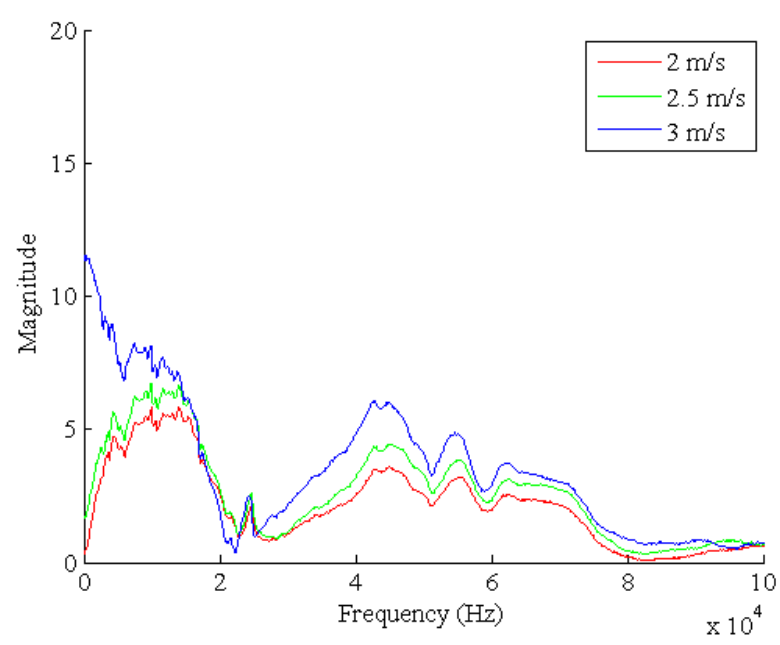

Fig. 10 Power spectra of $3 \mathrm{~mm}$ ball bearing impact signal

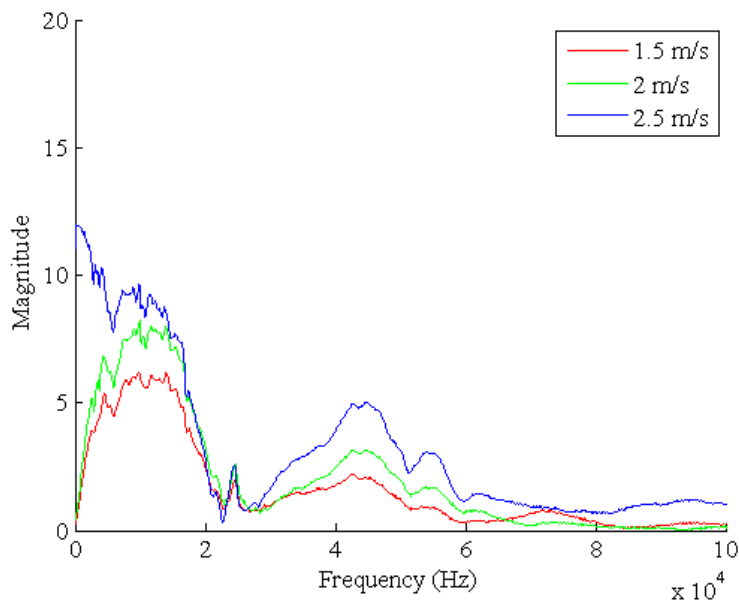

Fig. 11 Power spectra of $3.5 \mathrm{~mm}$ ball bearing impact signal 


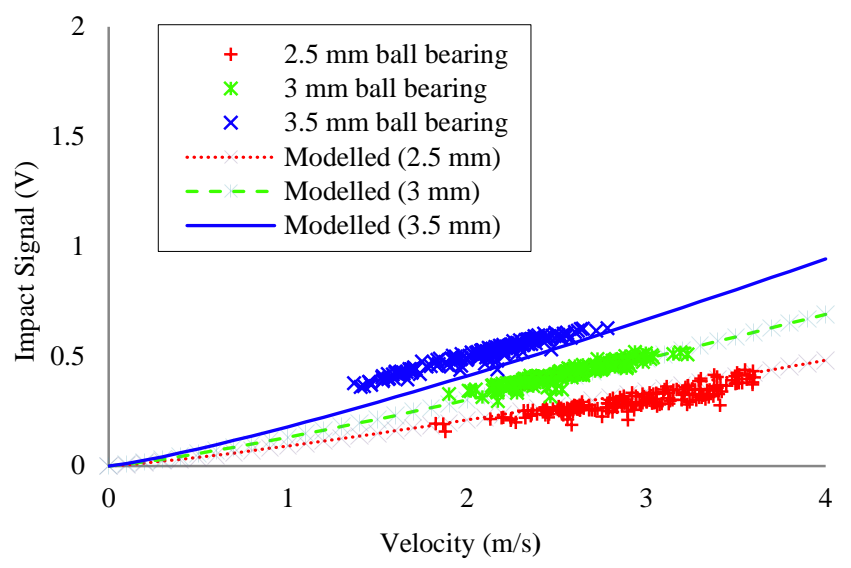

Fig. 12 Magnitude of dominant peak of ball bearing impact (with bandpass filter)

The same experiment was repeated again 200 times for all three ball bearing diameters using the bandpass filter. Fig. 12 shows the peak amplitude of the filtered impact signals. Fig. 12 also shows the calculated signal magnitude using the mechanical characteristics of the waveguide and the Delrin ball bearings and a range of impact velocities.

The signal output from the impact sensor agrees well with the calculated values using (8). However, the results from the $2.5 \mathrm{~mm}$ impacts show a higher deviation compared to the other two. This is believed to be due to the fact that, even though the guiding tube is centred above impact sensor waveguide, some of the $2.5 \mathrm{~mm}$ ball bearings may not be impacting the centre of the waveguide. Also for higher impact forces the impact sensor output is greater than the calculated values (3.5 mm ball bearing impacts). This is possibly due to the higher impact force being able to overcome the adhesive effect between the rubber and the waveguide [14]. This indicates that for a spherical particle, if the particle velocity is known as well as the mechanical characteristic of the particle, particle size can be determined via impact analysis using the piezoelectric impact sensor.

\section{CONCLUSIONS}

A new impact sensor using custom fabricated PVDF transducers has been designed, constructed and tested. Through analysis of the frequency component of the sensor output during impact it has been determined that selective filtering of frequency components of the impact signal has an effect on reducing the deviation of the peak magnitude of the impact signal.

The comparison between the calculated output signal values and the sensor outputs obtained during the experimentation agree well. This outcome indicates that, for a spherical particle, the piezoelectric impact sensor is capable of determining the size of the particle through impact analysis, assuming that the mechanical properties of the particle and particle velocity are known. For utilization of this sensor on a bulk solid conveying system the particle velocity would have to be independently measured using another sensing method such as electrostatic sensors [7].
The impact sensor proposed has a very small active sensing area, making it ideal for online particle size measurement on a pneumatic conveying system since the small active sensing area reduces the chance of simultaneous impacts of multiple particles occurring.

\section{REFERENCES}

[1] Y. Yan, "Guide to the Flow Measurement of Particulate Solids in Pipelines”, Powder Handling \& Processing, vol. 13, no. 4, October/November 2001.

[2] R. M. Carter, Y. Yan, P. Lee "On-line Nonintrusive Measurement of Particle Size Distribution through Digital Imaging”, IEEE Transactions on Instrumentation and Measurement, vol. 55, no. 6, pp. 2034 - 2038, 2006

[3] L. Gao, Y. Yan, G. Lu, "Contour-based Image Segmentation for On-line Size Distribution Measurement of Pneumatically Conveyed Particles", Instrumentation and Measurement Technology Conference, pp. 285-289, Hangzhou, China, 10-12 May 2011.

[4] J. Q. Zhang and Y. Yan, “On-line Continuous Measurement of Particle Size Using Electrostatic Sensors”, Instrumentation and Measurement Conference, pp. 877-880, Vail, CO, USA, 20-22 May 2003.

[5] B. M. Meunier, P. M. Watts, J. S. Marshall, R. L. Dechene, W. Du, R. E. Newton, "Vibration Sensor for Particle Concentration Measurement in Pneumatic Pipeline Flows”, Measurement Science and Technology, vol. 2, no. 12, 2010.

[6] L. Gao, Y. Yan, R. M. Carter, D. Sun, P. Lee, C. Xu, "On-line Particle Sizing of Pneumatically Conveyed Biomass Particles Using Piezoelectric Sensors”, FUEL, vol. 113, pp. 810 - 816, November 2013.

[7] J. R. Coombes and Y. Yan, "Experimental investigations into the flow characteristics of pneumatically conveyed biomass using an electrostatic cross sectional sensor array”, FUEL, vol. 151, pp. 11 - 20, July 2015

[8] Y. Hu, L. Wang, X. Huang, X. Qian, L. Gao, Y. Yan, “On-line Sizing of Pneumatically Conveyed Particles Through Acoustic Emission Detection and Signal Analysis”, IEEE Transactions on Instrumentation and Measurement, vol. 64, no 5, pp. 1100 - 1109, May 2015.

[9] P. Müller, M. Trüe, R. Böttcher, J. Tomas, “Acoustic evaluation of the impact of moist spherical granules and glass beads", Powder Technology, vol. 278, pp. 138 - 149, July 2015.

[10] H. Hertz, Über die berührung fester elastischer Körper (On the contac of rigid elastic solids). In: Miscellaneous Papers. Jones and Schott, Editors, J. reine und angewandte Mathematik 92, Macmillan, London (1896), p. 156 English translation: Hertz, H.

[11] S. Antonyuk, S. Heinrich, J. Tomas, N. G. Deen, M. S. van Buijtenen and J. A. M. Kuipers, "Energy absorption during compression and impact of dry elastic-plastic spherical granules”, Granular Matter, vol. 12, no 1, pp. 15 - 47, 2010.

[12] K. Uchino, “Advance Piezoelectric Materials”, Woodhead Publishing, Cambridge, UK, ISBN 978-1-84569-534-7.

[13] Measurement Specialties Inc. "Piezo Film Sensors Technical Manual”, REV B 02 APR 99.

[14] N.K. Myshkin, M.I. Petrokovets and A.V. Kovalev, "Tribology of polymers: Adhesion, friction, wear, and mass-transfer”, Tribology International, vol. 38, no 11, pp. 910 - 921. 